\title{
Série histórica dos conceitos do Enade em Odontologia: houve mudanças ao longo de três ciclos avaliativos?
}

\author{
Mariele Silva de Azevedo*; Thayse Gonzaga Gomes*; Rafael Lucas Sales Barbosa*; Allan Vinícius \\ Martins de Barros**; Andrea Cristina Barbosa da Silva ${ }^{* * *}$; Pedro Henrique Sette-de-Souza ${ }^{* * * *}$
}

\author{
Estudante de Graduação em Odontologia na Universidade \\ Estadual da Paraíba \\ ** Estudante de Graduação em Odontologia da Universidade de \\ Pernambuco \\ *** Docente, Universidade Estadual da Paraíba \\ **** Docente, Universidade Estadual da Paraíba e Universidade de \\ Pernambuco
}

Recebido em 28/09/2016. Aprovado em 23/01/2017.

\begin{abstract}
RESUMO
O Sistema Nacional de Avaliação em Educação Superior (SINAES) é responsável por avaliar as Instituições de Educação Superior (IES) públicas e privadas, sendo o Exame Nacional de Desempenho Acadêmico (Enade) um de seus eixos avaliativos, cuja finalidade é aferir o perfil e o desempenho dos alunos universitários e as características do curso ofertado. O Enade é realizado pelos concluintes do curso e se repete a cada três anos para a mesma área de conhecimento. O objetivo desse trabalho foi avaliar uma série histórica dos conceitos do Enade dos cursos de graduação em Odontologia de IES públicas e privadas do Brasil a fim de verificar as mudanças ocorridas ao longo de três ciclos avaliativos. Para tanto, buscou-se nos relatórios disponibilizados pelo INEP os conceitos Enade de todos os cursos avaliados na área de odontologia, nos anos de 2007, 2010 e 2013. Os dados foram submetidos à análise descritiva por meio da média e desvio-padrão dos conceitos e categorizados de acordo com a Unidade da Federação e, posteriormente, agrupados nas macrorregiões, de acordo com a categoria administrativa. Apesar das diferenças regionais quanto à concentração de cursos de Odontologia, os cursos de IES públicas foram mais bem avaliados no Enade do que os de IES privadas, independentemente da região. Os cursos de odontologia de IES pública são mais bem avaliados do que os de IES privadas, no Enade, independente da região. Somente os estados do Rio Grande do Norte e do Ceará tem todas as IES públicas com conceito 5. Descritores: Educação em Odontologia. Avaliação Educacional. Educação Superior.
\end{abstract}

\section{INTRODUÇÃO}

A avaliação de ensino superior está associada diretamente a diversos grupos e instituições que envolvem governos, estudantes, docentes, pesquisadores e a sociedade de forma geral. Cada um desses grupos possui visões distintas quanto ao sistema de avaliação, e a qualidade sobre a educação superior $^{1}$. A avaliação tem por objetivo gerar e organizar reformas educacionais para que mudanças nos currículos sejam realizadas, seja nas metodologias de ensino, na formação superior, 
nos modelos institucionais, na pesquisa e na responsabilidade social como um todo ${ }^{2}$.

Em meados dos anos 1990, o Ministério da Educação (MEC) criou o sistema de avaliação para ensino superior do Brasil. Assim, a partir da Lei $n^{\circ}$. 9.131, teve início o Exame Nacional de Cursos (ENC), conhecido como "Provão", aplicado para alunos concluintes de campos e áreas de conhecimento pré-estabelecidos. O ENC foi atingindo aos poucos todos os cursos de nível superior, passando de três áreas de conhecimento em 1996 para 26 em 2003. Contudo, apesar do pioneirismo, o Provão não indicou um padrão e escores mínimos para qualificar os cursos, além do mais recebeu muitas críticas por parte de acadêmicos e profissionais de avaliação, necessitando de mudanças ${ }^{2,3}$.

O objetivo do ENC situava-se no desempenho dos alunos, deixando de apontar alguns aspectos fundamentais, como os valores e os conhecimentos adquiridos ao longo da formação relacionados à carreira profissional, compromisso social e ética ${ }^{1}$. As provas eram aplicadas anualmente, possuíam caráter obrigatório, com os resultados publicados na mídia em forma de rankings, sendo por isso bastante questionado pela comunidade acadêmica ${ }^{4}$.

Dessa forma, avaliar o desempenho dos universitários tornou-se alvo de interesses externos, ocasionando a disputa entre aquelas que não se enquadrassem nos melhores índices de desempenho. Então, começaram a surgir os "mercados na educação superior" em que as instituições concorriam entre si na busca pela maior quantidade de alunos e pela melhor posição no ranking, mesmo que para isso fossem necessários cursinhos preparatórios para os exames e privilegiar os conteúdos das provas nos planos de ensino das disciplinas ${ }^{1}$.

Diante das críticas ao ENC, em 2004 criouse uma nova forma de avaliar o desempenho dos discentes, o Sistema Nacional de Avaliação em Educação Superior (SINAES), instituído pela Lei $\mathrm{n}^{\mathrm{o}} .10 .861$, de 14 de abril de 2004, que apresentou inovações para corrigir as deficiências do $\mathrm{ENC}^{5}$. O SINAES é responsável por avaliar as Instituições de Educação Superior (IES) públicas e privadas por meio de três eixos: Avaliação das IES, dos Cursos de Graduação e Exame Nacional de Desempenho Acadêmico (Enade), cuja finalidade é aferir o desempenho dos estudantes universitários com ênfase nas Diretrizes Curriculares Nacionais (DCN) de cada curso superior, com foco nas habilidades e competências exigidas pela formação profissional. Os exames que compõem a avaliação são a prova, um questionário socioeconômico para o estudante e um para os coordenadores do curso, sendo que o primeiro irá demonstrar o perfil do corpo discente, conhecer a opinião dos estudantes, reunir informações sobre as condições de ensino e metodologias de ensinoaprendizagem. No segundo, são verificadas as características positivas e negativas do curso ofertado ${ }^{4}$.

O Enade é realizado a cada três anos para os cursos de bacharelado e licenciatura da mesma área do conhecimento. O Instituto Nacional de Estudos e Pesquisas Educacionais Anísio Teixeira (INEP) é responsável pela sua administração, mediante supervisão da Comissão Nacional de Avaliação da Educação Superior (CONAES). Anteriormente, a prova era igualmente aplicada com os mesmos conteúdos para ingressantes e concluintes. Entretanto, nos últimos anos, somente os concluintes, ou seja, aqueles que integralizaram $80 \%$ da carga horária do curso, realizam a prova ${ }^{6}$. O resultado é categorizado numa escala de cinco níveis crescentes, no qual 1 é o pior resultado e 5 o melhor ${ }^{1}$.

Assim, o objetivo desse estudo foi analisar uma série histórica dos conceitos do Enade dos 
cursos de graduação em Odontologia das IES públicas e privadas do Brasil, a fim de verificar se ocorreram mudanças ao longo de três ciclos avaliativos consecutivos.

\section{METODOLOGIA}

Em um primeiro momento, buscou-se nos relatórios disponibilizados pelo INEP, em seu sítio, os conceitos Enade de todos os cursos avaliados na área de Odontologia nos anos de 2007, 2010 e 2013. A partir de então, criou-se um banco de dados, incluindo a localização da IES (Unidade da Federação/UF e Macror-região), a categoria administrativa (pública ou privada) e os conceitos dos Enade dos três ciclos. Os dados foram submetidos à análise descritiva por meio da média e desvio-padrão dos conceitos e categorizados, inicialmente, de acordo com a UF e, posteriormente, agrupados nas macrorregiões, de acordo com a categoria administrativa.

\section{RESULTADOS E DISCUSSÃO}

Os resultados da média (desvio-padrão) da série histórica do Enade (2007 a 2013) por macrorregião brasileira e por UF são mostrados na tabela 1 .

$\mathrm{Na}$ região Norte, as escolas públicas de Odontologia caíram de conceito ao longo do tempo, ao contrário das IES privadas, que tiveram conceito aumentado. Contudo, ainda se observa que a média destas é inferior à das IES públicas na região, ficando aquém da média nacional e da média das demais regiões.

Além disso, é possível observar que ainda há unidades da federação que não possuem IES públicas que oferecem o curso de Odontologia (Acre, Amapá, Roraima, Rondônia e Mato Grosso), sendo quase todos concentrados na região Norte do país. Há cinco anos existiam mais de 220.000 cirurgiões-dentistas (CDs) distribuídos no território brasileiro, no entanto apenas $3,73 \% \quad(1: 1723$ habitantes $)$ estavam localizados na região Norte, ao passo que a região Sudeste concentrava 58,39\% dos profissionais (1:597 habitantes). Considerando que a Organização Mundial de Saúde (OMS) recomenda a proporção de $1 \mathrm{CD}$ para 1500 habitantes, esses dados demonstram o quanto a distribuição é desproporcional entre as regiões brasileiras $^{7}$, o que certamente está sendo influenciando pelo número de cursos de Odontologia nessas regiões.

A região Nordeste apresentou a maior média no Enade 2013 dentre as IES públicas, sendo seguida pela região Centro-Oeste. Destacam-se os estados do Rio Grande do Norte e o Ceará por serem os únicos a apresentarem o conceito máximo (5) das IES públicas. Os conceitos das IES privadas decaíram ao longo dos três anos de aplicação do exame.

No Centro-Oeste, observa-se o segundo melhor conceito das IES públicas, as quais foram diminuindo ao longo do período do estudo. Enquanto as IES privadas apresentaram um pequeno aumento, mas ainda abaixo da média ideal $\geq 3$.

$\mathrm{Na}$ região Sudeste também ocorreu diminuição nos conceitos das IES públicas. Quanto às IES privadas, houve um aumento de 2007 a 2010 e diminuição em 2013. Nessa região existe a maior quantidade de cursos de Odontologia ofertados, porém pelos resultados do Enade não se observa efeito positivo desta concorrência.

Na região Sul houve diminuição no conceito tanto nas IES públicas quanto nas privadas. A segunda maior concentração dos cursos de Odontologia ofertados está nesta região. Mesmo que os conceitos do Enade das IES públicas tenham mantido uma média ideal > 4, estas foram diminuindo ao longo do período em estudo.

Observa-se também em nível nacional que as IES públicas obtiveram conceitos que se sobressaíram em relação às privadas, o que remete à necessidade de reflexão quanto à qualidade do 
Tabela 1: Média e desvio-padrão dos conceitos do Enade, por Unidade da Federação e Região, de acordo com a natureza da IES.

\begin{tabular}{|c|c|c|c|c|c|c|}
\hline \multirow[t]{2}{*}{ Região/UF } & \multicolumn{2}{|c|}{ ENADE 2007} & \multicolumn{2}{|c|}{ ENADE 2010} & \multicolumn{2}{|c|}{ ENADE 2013} \\
\hline & Públicas & Privadas & Públicas & Privadas & Públicas & Privadas \\
\hline Norte & $3,33(1,15)$ & $2,00(0,89)$ & $2,75(1,25)$ & $2,18(0,87)$ & $2,75(1,25)$ & $2,35(0,84)$ \\
\hline $\mathrm{AC}$ & SA & SA & SA & SA & SA & $3,00(*)$ \\
\hline $\mathrm{AM}$ & $4,00(*)$ & $1,67(0,57)$ & $3,50(0,70)$ & $2,40(1,14)$ & $3,50(0,70)$ & $2,20(1,09)$ \\
\hline $\mathrm{AP}$ & SA & SA & SA & SA & SA & $2,00(*)$ \\
\hline PA & $4,00(*)$ & $3,00(*)$ & $3,00(*)$ & $2,00(*)$ & $3,00(*)$ & $3,00(*)$ \\
\hline RO & SA & $2,00(1,41)$ & SA & $2,50(0,70)$ & SA & $2,33(0,58)$ \\
\hline $\mathrm{RR}$ & SA & SA & SA & $2,00(*)$ & SA & $3,00(*)$ \\
\hline TO & $2,00(*)$ & SA & $1,00(*)$ & $1,50(0,70)$ & $1,00(*)$ & $2,00(1,41)$ \\
\hline Nordeste & $4,00(0,42)$ & $2,91(0,67)$ & $3,67(0,81)$ & $2,69(0,63)$ & $4,42(0,65)$ & $2,57(0,65)$ \\
\hline $\mathrm{AL}$ & $4,00(*)$ & $3,00(*)$ & $4,00(*)$ & $3,00(*)$ & $4,00(*)$ & $2,00(*)$ \\
\hline BA & $4,00(0,00)$ & $3,00(1,00)$ & $3,00(1,00)$ & $2,67(0,58)$ & $4,50(0,70)$ & $3,33(0,58)$ \\
\hline $\mathrm{CE}$ & $4,00(*)$ & $4,00(*)$ & $4,00(1,41)$ & $3,50(0,70)$ & $5,00(0,00)$ & $2,50(0,70)$ \\
\hline MA & $4,00(*)$ & $2,00(0,00)$ & $4,00(*)$ & $2,50(0,70)$ & SA & $3,00(*)$ \\
\hline PB & $4,00(0,00)$ & SA & $3,50(0,70)$ & SA & $4,33(0,58)$ & $2,00(0,00)$ \\
\hline PE & $4,00(0,00)$ & $3,00(0,00)$ & $4,00(0,00)$ & $2,50(0,70)$ & $3,50(0,70)$ & $2,50(0,70)$ \\
\hline PI & $3,50(0,70)$ & $3,00(0,00)$ & $3,00(0,00)$ & $2,00(0,00)$ & $4,50(0,70)$ & $2,00(0,00)$ \\
\hline $\mathrm{RN}$ & $5,00(*)$ & $3,00(*)$ & $4,50(0,70)$ & $3,00(*)$ & $5,00(0,00)$ & $3,00(*)$ \\
\hline SE & $5,00(*)$ & $3,00(*)$ & $4,00(*)$ & $3,00(*)$ & $4,00(*)$ & $2,00(*)$ \\
\hline Centro-Oeste & $4,67(0,57)$ & $2,14(0,69)$ & $4,00(0,00)$ & $2,90(0,74)$ & $4,33(0,57)$ & $2,82(1,17)$ \\
\hline DF & $4,00(*)$ & $1,67(0,57)$ & $4,00(*)$ & $2,67(0,57)$ & $5,00(*)$ & $3,00(0,00)$ \\
\hline GO & $5,00(*)$ & $2,00(0,00)$ & $4,00(*)$ & $3,00(1,41)$ & $4,00(*)$ & $2,33(1,52)$ \\
\hline MS & $5,00(*)$ & SA & $4,00(*)$ & $3,50(0,71)$ & $4,00(*)$ & $3,00(0,00)$ \\
\hline MT & SA & $3,00(0,00)$ & SA & $2,67(0,58)$ & SA & $3,00(2,00)$ \\
\hline Sudeste & $4,25(0,68)$ & $2,68(0,66)$ & $3,67(0,59)$ & $2,88(0,89)$ & $3,41(0,79)$ & $2,71(0,88)$ \\
\hline ES & $5,00(*)$ & 3,00 & $4,00(*)$ & $3,00(0$ & $4,00(*)$ & $2,67(0,58)$ \\
\hline MG & $4,33(0,51)$ & $2,63(0$, & $4,00(0,00)$ & $2,67(0,72)$ & $3,67(0,51)$ & $2,67(0,48)$ \\
\hline RJ & $4,33(0,58)$ & $2,64(0,74)$ & $3,50(0,57)$ & $2,92(0,95)$ & $3,00(1,15)$ & $2,61(0,77)$ \\
\hline SP & $4,00(0,89)$ & $2,70(0,64)$ & $3,43(0,79)$ & $2,97(0,98)$ & $3,33(0,82)$ & $2,78(1,09)$ \\
\hline Sul & $4,40(0,84)$ & $3,21(0,78)$ & $4,180,60)$ & $2,95(0,60)$ & $4,10(0,56)$ & $2,73(0,65)$ \\
\hline PR & $4,60(0,54)$ & $2,88(0,60)$ & $4,20(0,44)$ & $2,67(0,50)$ & $4,00(0,00)$ & $2,55(0,52)$ \\
\hline RS & $5,00(0,00)$ & $3,50(1,05)$ & $4,67(0,57)$ & $3,28(0,48)$ & $4,67(0,57)$ & $2,71(0,76)$ \\
\hline $\mathrm{SC}$ & $3,67(1,15)$ & $3,50(0,57)$ & $3,67(0,57)$ & $3,00(0,81)$ & $3,67(0,57)$ & $3,33(0,57)$ \\
\hline Brasil & $4,20(0,73)$ & $2,74(1,78)$ & $3,73(0,77)$ & $2,81(1,75)$ & $3,83(0,88)$ & $2,66(1,86)$ \\
\hline
\end{tabular}

$\mathrm{SA}=$ Sem avaliação no Enade; * = sem desvio-padrão (apenas uma instituição no grupo).

ensino nestes. O Brasil possui um dos maiores números mundiais de CDs, e o número de graduados está em constante aumento.

Portanto, por meio das informações do SINAES e do Enade, os governos e responsáveis pelas IES devem assumir a responsabilidade na formação e competências para atuar na solução dos problemas de saúde bucal do país principalmente com base nas DCNs e nos currículos integrados. O objetivo é que o discente de Odontologia adquira excelência técnica associada à postura criteriosa e coerente, para saber o que fazer, quando fazer, como fazer, onde fazer, por que e para quem fazer, o que significa estabelecer um diagnóstico correto e amplo da situação em benefício da sociedade ${ }^{8}$.

Vale salientar que mesmo com os avanços advindos por meio da avaliação feita por meio do Enade, esse sistema ainda é bastante criticado, 
principalmente porque os escores atribuídos não refletem, de fato, a capacidade técnico/profissional do egresso.

\section{CONCLUSÕES}

Os cursos de Odontologia de IES públicas obtiveram melhores conceitos no Enade em comparação aos de IES privadas, independente da região. Somente os estados do Rio Grande do Norte e do Ceará tem todas as IES públicas com conceito 5.

\section{ABSTRACT \\ Historical series of Enade grades in Dentistry: were there changes throughout three evaluative cycles?}

The National System for Evaluation in Higher Education (SINAES) is responsible for evaluating Higher Education Institutions (IES) in Brazil and the National Student Proficiency Exam (Enade) is one of its evaluative axis which aims to assess the profile and the performance of the university students and the technical features of the available courses. The Enade is performed once in every three years for each field of knowledge by those students who are finishing the course. The purpose of this study was to evaluate a historical series of the Enade grades for Dental undergraduate courses of public and private higher education institutions in Brazil to check the variations over three evaluative cycles. For this, Enade grades for all the Dental courses evaluated in the years of 2007, 2010 and 2013 available in the reports provided by INEP were analyzed. The data were submitted to a descriptive analysis by the mean and standard deviation of the grades and categorized according to the Federated Unit and then put together in macroregions and according to its administrative category. Despite the difference in the concentration of the number of courses between the macroregions, those from the public higher education institutions have shown better grades in all situations. The dentistry courses of public HEI reached better grades than those of private HEI, in the Enade, independent of the region. Only the states of Rio Grande do Norte and Ceará have all public HEIs with a grade of 5 .
Descriptors: Dentistry Education. Educational Evaluation. College Education.

\section{REFERÊECIAS}

1. Bertolin JSG, Marcon T. O (des) entendimento de qualidade na educação superior brasileira - Das quimeras do provão e do ENADE à realidade do capital cultural dos estudantes. Avaliação. 2015;20(1):105-22.

2. Sobrinho JD. Avaliações e transformações da educação superior brasileira (1995-2009): do provão ao SINAES. Avaliação. 2010;15(1):195-224.

3. Verhine RE, Dantas LMV, Soares JFS. Do provão ao ENADE: Uma análise comparativa dos exames nacionais utilizados no Ensino Superior Brasileiro. Ensaio: Aval Pol Públ Educ. 2006;14 (52):291-310.

4. Santos J, Silva ML. O exame nacional de desempenho (ENADE): concepções e proposições na percepção discente. Horizonte Científico. 2014;8(1): 1-23.

5. Paiva GS. Avaliação do desempenho dos estudantes da educação superior: a questão da equidade e obrigatoriedade no provão e no ENADE. Ensaio: Aval Pol Públ Educ. 2008;16(58):31-46.

6. Tumolo LMS. O ENADE como processo de autoavaliação dos cursos de graduação e da instituição. Cad Acad. 2010;2(2):1-14.

7. Paranhos LR, Ricci IV, Scanavini MA, Bérzin F, Ramos AL. Análise do mercado de trabalho odontológico na Região Sul do Brasil. Rev Fed Odontol. 2009;14(1):7-13.

8. Gabriel M, Tanaka EE. Formação profissional em Odontologia: percepção discente da interação curricular. Rev ABENO. 2011;11(1):19-22.

Correspondência para:

Pedro Henrique Sette-de-Souza

e-mail: pedro.souza@upe.br

Rua Gumercindo Cavalcante, 420

56512-200 São Cristovão, Arcoverde/PE 